\title{
THE MANAGEMENT OF THE BLEEDING TONSIL
}

\author{
By J. Ivor Griffiths, M.S., F.R.C.S.
}

Laryngologist, British Post-Graduate School. Consulting Surgeon and Laryngologist, Acton Hospital

In this short discourse we shall deal from a practical point of view with those problems which are associated with excessive bleeding connected with the operation for the removal of tonsils and adenoids. The operation is undertaken daily by the thousand without there being, as a rule, any thought or anticipation of the possibility of bleeding, and if this unfortunate event arises it is dealt with in a dramatic but often haphazard manner at that time. So little can be done to prevent its onset that we rely almost entirely on the clotting powers of the blood to save what might seem to be a certain catastrophe were we to stop and consider the chances. After all, the tonsils and adenoids have a substantial blood supply, large areas are laid bare by the operation, and the cut surfaces are left widely exposed in the pharynx and nasopharynx to heal by Nature's best intention. Sloughs must form under far from ideal conditions and must become infected with the normal flora of the mouth and nose, and it is recognized by all that it is infection beyond all other causes which predisposes to secondary haemorrhage.

The first step, therefore, is to question ourselves as to whether there is any precaution that can be taken to prevent haemorrhage either during or following the operation. In the average case nothing of any real value has been found, but if there are any suspicions raised as to the slowness of clotting (and any one member of the family will be able to supply this information), it is well to have the 'clotting time' calculated beforehand. A true haemophylic is, of course, no candidate for the operation, but many cases will show a retarded clotting time. If so, it would be well to treat such a case with intramuscular injections of calcium (calcium gluconate 10 per cent. sol. 5-10 c.c. daily) for a few days before the operation and combine this therapy with injections of vitamin $\mathrm{K}$ also.

Cases of recent throat infections should be given adequate time for local inflammation with its engorging effect on the vessels to have subsided completely. A minimum period of six weeks from the acute infection should be insisted on, otherwise an excessive amount of bleeding at the time of operation prolongs the recovery of the patient from two to three weeks to as many months, or longer.

\section{During the Operation}

There must remain one axiom in this operation as in all others, that all large vessels must be clamped and ligatured, and small vesst ls allowed to close by their own inherent ability of retraction, thus allowing a small clot to form on the surface.

It is a matter of choice what material is used-catgut or silk, and whether the vessels be tied or sewn. Most surgeons prefer silk because a more secure knot can be tied and it is less likely to be disturbed by the vomiting or strain on the throat which invariably occurs during the stage of restlessness in recovery from the anaesthetic.

If these measures fail-and the more often will they fail in the less experienced handsthe surgeon will be obliged to pack the tonsillar fossae with gauze and to stitch the pillars together, leaving the packs in situ for two or three days.

All sorts of devices have been tried to stop the so-called oozing tonsil, which is mostly due to bleeding from a vein which has been cut longitudinally and does not appear to stop either by pressure or by forming an adherent clot. Snake venom applied on a pledget of cotton wool, foreign blood sera, adrenalin packs - the least satisfactory of all, and in the writer's opinion a dangerous drug to use in such conditions-styptics of various kinds, ferric chloride, alum and Friars balsam, and the latest and probably the best of all, thrombin, are all used. None of these methods of attack should be necessary while the patient is anaesthetized although they may be useful and worthy of trial when the patient has recovered from the anaesthetic and is suffering from reactionary bleeding.

\section{After the Operation}

Reactionary bleeding is the main concern of all surgeons and is liable to happen to any case 
however carefully the operation has been conducted; though, naturally, it becomes less common in the hands of the more experienced.

This type of bleeding occurs within the first 24 hours of the operation, and quite often during the stage of recovery from the anaesthetic. The management of such a case and correct judgment in deciding whether or not the patient is to be reanaesthetized is often difficult, and may be the difference between the life or death of the patient, the difference between a rapid recovery or long complications due to aspiration of blood clots during the reanaesthetization, pneumonia and lung abscesses. On such decision also lies the difference between the necessity of giving the patient a long draught of water to drink or all the lightning paraphernalia of a blood transfusion. The question is what to do and how and when to do it.

Haemorrhage from a tonsil or adenoid bed must be stopped whenever possible before giving blood transfusions. The surgeon or the house surgeon is called by the ward sister and told that the patient is either spitting a lot of blood or has been vomiting large amounts of black blood from the stomach. The picture is always the same-disturbed relatives, a most anxious, often restless and hot, patient who bears a white sweating countenance, with a rapid pulse and a low blood pressure. It is comforting to realize that the picture usually exaggerates the real loss of blood, because the amount vomited is not a true record of the volume of blood lost as it is mixed with stomach contents and added to by a massive out-pouring of fluid from the stomach, and that the blood pressure is lowered by the presence of blood in the stomach. Once all the stale blood has been ejected from the stomach either by natural means or following the time-honoured practice of giving a draught of bicarbonate of soda then the blood pressure immediately improves. Stop the bleeding, empty the stomach and within an hour the patient feels and looks a different being. This is positive proof that the given picture is not due entirely to loss of blood.

There are certain steps to be taken immediately the crisis has been recognized; it cannot be overemphasized that failure to perform these leads to death in not a few cases.
The enquiries which follow have been the source of great distress to relatives, doctors and hospitals, examples of which we read about from time to time in the columns of the daily Press.

The first step is to approach the patient with confidence. $\mathrm{He}$ is in dire need of it himself and he must be told quietly yet firmly to keep still even if painful and unpleasant manipulations are to follow.

The patient should be on his side so that he can spit into a bowl without jumping up and down every time the pharynx fills with blood or a clot tends to fall down the pharynx. The position of lateral decubitus is of paramount importance.

The next requirement is that the surgeon must have a good headlight or mirror in order to have both hands free.

Thirdly, the tongue must be completely depiessed and a full view of the pharynx obtained. Now is the time to assess the bleeding-which side and how much. In a large proportion of cases the cause will be evident-to wit, a large massive clot in one or other fossae with blood trickling from the deep part of the clot. Every bit of this clot must be removed because it is this superficial clot, large as it is, which is itself preventing the retraction of the underlying vessel. Removal of this clot, a little patience and the calming of the patient by administrating morphia if necessary will be all that is necessary in 8o-9o per cent. of cases if this manoeuvre is carried out properly. 'If, after removing the clot, we find no definite artery or vein visibly bleeding then the commoncst site for bleeding is the cut edge of the tongue which now overhangs the bleeding point and prevents direct access. A firm pack using a styptic or film of fibrin will sometimes help, and in this particular type of haemorrhage the patient should be made to bite some object like the handle of a tooth-brush in order to prevent the repeated act of swallowing encouraged by the blood trickling into the pharynx. Unless there is a fairly quick response to these manoeuvres this type of case, because of the peculiar inaccessibility of the bleeding vessel, will be better anaesthetized and the bleeding point searched for and, when found, ligatured.

Minor oozing from the fossa will usually stop 
after removing the clot, but if it does not then a firm pack of cotton wool or gauze swab protected by an attached thread plus the fibrin swab or snake venom will stop almost all of the cases in this group.

If it is decided to reanaesthetize the patient this should be done in the operating theatre, and the only trouble to anticipate is inspiration of blood clot during the induction period with its obvious immediate and post-operative dangers to the lung. Bronchopneumonia, collapse of the lung and lung abscess are sequels which none can afford to ignore.

The induction should be rapid, the new intravenous drugs such as pentothal being a great help. The Boyle Davies gag should be introduced at the first opportunity, the pharynx and larynx kept free of blood by suction while the fuller anaesthesia is being obtained. The vessel should be searched for and, when found, clamped and then securely tied with a silk stitch.

Adenoidal haemorrhage of any consequence is rare, but occurs from time to time. The treatment is the same. The importance of removing any large clot present cannot be overemphasized, but if the bleeding is persistent the nasopharynx should be packed with gauze impregnated with fibrin, without an anaesthetic if possible. Here, again, it is all important to recognize the area from which the blood is oozing. One has been called to see a moribund patient who had had two blood transfusions and an anaesthetic yet, in spite of this, the fact that the bleeding was originating in the nasopharynx had not been recognized. In this case the bleeding stopped once the clot was removed, thereby showing that neither the blood transfusions nor the risk of the anatsthetic had been either necessary or justified.

Secondary haemorrhage. Such bleeding occurs from the fifth to seventh day, but has been known to occur up to the twelfth day after operation. The bleeding is due to separation of sloughs and is more common in badly traumatized throats. The bleeding is usually from one small pinpoint which is usually visible. Removal of the slough and slight pressure of a swab plus fibrin will stop the bleeding. The patient has had time to recover from the operation and, therefore, presents a less complicated clinical picture. $\mathrm{He}$ usually notices the blood and spits it out, ànd therefore one is less likely to be misled by the swallowed blood, which is usually negligible.

It will have been noted that I have not recommended the use of the tonsillar clamp because this instrument causes excessive pain and is so often unsuccessful. It is of no use for the bleeding vessel concealed under the base of the tongue, while vessels amenable to pressure in the tonsillar fossa can be dealt with in a more precise and certain manner by direct pressure or ligature.

If a bleeding vessel is seen and, of course, in most cases it can, then it will not be difficult to clip the vessel with a long pair of curved artery forceps. The forceps can be left in situ for a short period without pain ; the pain is caused at the time of clipping the tissue, so one should be very certain of one's aim. A delicate pair of hands might well put on a ligature and settle the problem. All this can and should be done without recourse to an anaesthetic ; but there are exceptional patients and circumstances.

But before concluding it is as well to remind ourselves that after an operation for removal of tonsils and adenoids, haemorrhage may take place from some other regions of the body, at the time not always easy to explain. If this fact is borne in mind it will prevent one jeopardizing the chances of the patient's recovery by giving unnecessary anaesthetics. Epistaxis from the usual source in the septum needs little momert. Epistaxis, however, occurred in one of my patients from the antrum. Plugging of the nose was of no avail. The antrum had to be punctured, the clot washed out with hot saline, and I tried the novel treatment of injecting 5 c.c. of haemostatic serum into the antrum and this proved successful therapeutically.

One interesting event happened to a patient of mine 12 days after tonsillectomy. The man was convalescing in a temporary Army bed which collapsed while he was asleep, and this resulted in his rupturing oesophageal veins and vomiting large amounts of blood. Gastric ulcers may bleed and give rise to conflicting diagnoses. And, finally, one has seen a boy who bled profusely from a rectal polypus on the same evening of the operation for tonsillectomy. 